\title{
DÚVIDAS MATERNAS NA ALTA HOSPITALAR DO RECÉM-NASCIDO
}

\section{MATERNAL DOUBTS ON NEWBORN DISCHARGE}

\author{
Gláucia Maria Nóbrega Rocha ${ }^{1}$ \\ Marielle Ribeiro Feitosa ${ }^{2}$ \\ Rhanna Emanuela Fontenele Lima de Carvalho ${ }^{3}$ \\ Regina Claúdia Melo Dodt ${ }^{4}$ \\ Maria Veraci de Oliveira Queiroz ${ }^{5}$ \\ Edna Maria Camelo Chaves Correio ${ }^{6}$
}

Resumo: Alta hospitalar é um momento importante para a mãe do recém-nascido prematuro, que irá assumir os cuidados com o filho. O objetivo dessa pesquisa é identificar as dúvidas apresentadas pelas mães de recém-nascidos prematuros em relação aos cuidados domiciliares no preparo para alta hospitalar. Os métodos utilizados foram embasados por um estudo descritivo, realizado com 30 mães que estavam com seus filhos com alta hospitalar programada, em uma unidade neonatal de um hospital público. O estudo se desenvolveu de maio a outubro de 2015. Os dados foram coletados por meio de um roteiro de entrevista semiestruturada. Os resultados demonstraram que a maioria das mães participantes da pesquisa informou não estar preparadas para cuidar do filho (60,1\%). As dúvidas mais relatadas foram o banho (60\%) e amamentação (56,7\%). Conclui-se que as mães não se sentiam seguras para prestar os cuidados de higienização e alimentação do bebê ao chegar ao domicilio.

Palavras-chave: Alta hospitalar; enfermagem neonatal; recém-nascido; unidade de terapia intensiva neonatal.

Abstract: Hospital discharge is an important time for the mother of the premature newborn, who will take care of the child. Objective: to identify the doubts presented by the mothers of preterm infants in relation to the home care in the preparation for hospital discharge. Methods: This is a descriptive study, conducted with 30 mothers who had a scheduled hospital discharge for their children, in a neonatal unit of a public hospital. The study was developed from May to October, 2015. The data were collected by a semistructured interview script. Results: Most of the mothers participating in the study reported not being prepared to take care of the child (60.1\%). The most frequently reported doubts were bathing (60\%) and breastfeeding (56.7\%). Conclusion: The mothers did not feel safe to provide the hygienic and feeding care to the baby when arriving at home

Keywords: Patient discharge; neonatal nursing; newborn infant; neonatal intensive care unit.

\section{INTRODUÇÃO}

O nascimento de um bebê prematuro gera muita expectativa e sentimento de medo, diante de um ser tão pequeno e frágil. Esse sentimento é vivenciado por toda a família, em especial pela mãe, que acompanha o seu filho durante o internamento. Sabe-se que muitas vezes, a mãe se depara com situações de agravo clínico do bebê, gerando dúvida, medo de perder o filho e ansiedade, além das dificuldades e das

\footnotetext{
${ }^{1}$ Mestra em Enfermagem, Universidade Federal do Ceará, Brasil. E-mail: glauciamnrocha@yahoo.com.br.

2 Mestra em Saúde da Família, Universidade Federal do Ceará, Brasil. E-mail: mariellerf@hotmail.com.

3 Professora Adjunta do Centro de Ciências da Saúde, Universidade Estadual do Ceará, Brasil. E-mail: rhannalima@gmail.com.

4 Professora Adjunta do Centro de Ciências da Saúde, Universidade Estadual do Ceará, Brasil. E-mail: reginadodt@gmail.com.

5 Professora Adjunta do Centro de Ciências da Saúde, Universidade Estadual do Ceará., Brasil. E-mail: veracioq@hotmail.com.

6 Professora Adjunta do Centro de Ciências da Saúde, Universidade Estadual do Ceará, Brasil. E-mail: ednacam3@gmail.com.
} 
repercussões que a hospitalização provoca no cotidiano da vida da família (RAPOPORT; PICCININI, 2011; SIQUEIRA; DIAS, 2011).

A alta hospitalar do recém-nascido prematuro é um momento que deve ser planejado com a participação da mãe e/ou família, pois diante da fragilidade, surgem o medo de cuidar, as dúvidas: o que poderá dificultar o cuidado prestado no domicílio (FROTA et al., 2013).

A prematuridade é uma realidade mundial que demanda a articulação de estratégias para o seu enfrentamento, sobretudo em relação à participação da família no contexto da assistência hospitalar (SANTOS et al., 2012), sendo considerado recém-nascido prematuro aquele que nasce com até 36 semanas e seis dias de gestação (BRASIL, 2011). A hospitalização representa uma experiência estressante, traumática e preocupante que afeta toda a estrutura familiar (MELO; SOUZA; PAULA, 2012).

O prematuro é uma criança de alto risco que para alcançar um desenvolvimento satisfatório, necessita de cuidados maternos específicos após a alta hospitalar e por um longo período de tempo (SCHIMIDT; HIGARASHI, 2012). Muitas vezes a mãe, diante da prematuridade, não se sente preparada para cuidar do filho. Isso gera a necessidade de identificação dos cuidados que possam ser orientados para 0 empoderamento da mãe no cuidado domiciliar.

Ressalta-se a importância do contexto familiar para o bebê prematuro, pois ele recebe os cuidados e influências para sua recuperação e seu desenvolvimento. Normalmente, as crianças egressas de uma unidade neonatal sofrem um impacto quando chegam ao domicílio, pois este geralmente não tem infraestrutura adequada, sem contar com o possível despreparo da mãe, que será sua principal cuidadora (FONSECA; MARCON, 2011; BOTÊLHO et al., 2012).

O cuidado centrado na família deve considerar os aspectos sociais, emocionais e psicológicos, levando também em conta as dificuldades que surgem ao longo da internação neonatal (COUTO; PRAÇA, 2012; FROTA et al., 2013). Cabem aos profissionais de saúde, buscar estratégias que favoreçam o preparo da alta e da continuidade do cuidado domiciliar.

Diante do exposto, o objetivo deste estudo é identificar as dúvidas apresentadas pelas mães de recém-nascidos prematuros em relação aos cuidados domiciliares no preparo para alta hospitalar.

\section{MÉTODO}

Trata-se de um estudo quantitativo, descritivo realizado em um hospital público de Fortaleza-Ceará-Brasil, referência no atendimento a recém-nascidos prematuros. $A$ população do estudo foi composta por mães cujos filhos prematuros encontravam-se 
internados na unidade neonatal, durante o período de coleta de dados. A amostra foi não probabilística, consecutiva, sendo composta por 30 mães.

O critério de inclusão foi o acompanhamento da mãe ao seu filho na unidade hospitalar até o momento da alta. Foram excluídas as mães dos bebês com solicitação de transferência para unidade Canguru.

Os dados foram coletados no período de maio a outubro de 2015, por meio de entrevista semiestruturada, com a finalidade de obter um diagnóstico situacional das mães em relação aos cuidados prestados durante o internamento na unidade neonatal e as principais dúvidas sobre os cuidados domiciliares que seriam realizados no domicilio. A pesquisadora, inicialmente, abordou as mães dentro da unidade neonatal e fez o convite para que participassem do estudo. As que aceitaram foram levadas para uma área de convivência, dentro da maternidade, para a realização da entrevista. A entrevista foi estruturada, sendo composta de duas partes. Na parte A foram colocados dados sócio demográficos e clínicos da mãe (questões de 1 a 15), seguida da parte $B$ com questões abertas que abordavam os cuidados como toque, o preparo para amamentação, as orientações para alta e as dúvidas em relação aos cuidados domiciliares.

Os dados foram organizados em planilhas eletrônicas do programa Microsoft Office Excel 2010, analisados por meio da estatística descritiva e apresentados em tabelas.

Conforme a Resolução 466/2012 que regulamenta a pesquisa com seres humanos, obteve-se a aprovação do Comitê de Ética em Pesquisa da Maternidade Escola Assis Chateaubriand, sob o parecer 1.052.985/2015. As mães adolescentes assinaram o termo de assentimento e as demais, o termo de consentimento livre esclarecido.

\section{RESULTADOS}

Os resultados da tabela 1 apontam que a maioria das entrevistadas apresentava idade entre 20 a 35 anos (66,7\%), casadas ou com união consensual $(60 \%)$, com média de 11 anos de estudo, do lar (26,6\%) e procedentes do interior $(46,7 \%)$.

Tabela 1 - Identificação socioeconômica da entrevistada. Fortaleza-CE, 2015.

\begin{tabular}{lccc}
\hline Variáveis maternas & $\mathrm{n}$ & $\%$ & $\mathrm{ME} \pm \mathrm{DP}$ \\
\hline $\begin{array}{l}\text { Idade materna (anos) } \\
14-19\end{array}$ & 6 & 20,0 & $26,33 \pm 7,66$ \\
\hline $20-35$ & 20 & 66,7 & \\
\hline$>35$ & 4 & 13,3 & \\
\hline Estado civil & & & \\
\hline Solteira & 12 & 40,0 & \\
\hline Casada & 10 & 33,3 & \\
\hline União consensual & 8 & 26,7 & \\
\hline
\end{tabular}




\begin{tabular}{lccl}
\hline Anos de estudo & & & \\
\hline $1-7$ & 3 & 10,0 & $11,23 \pm 2,44$ \\
\hline $8-11$ & 17 & 53,4 & \\
\hline$\geq 12$ & 10 & 36,6 & \\
\hline Ocupação & & & \\
\hline Do lar & 08 & 26,6 & \\
\hline Assalariada & 05 & 16,6 \\
\hline Autônoma & 04 & 13,3 \\
\hline Agricultora & 07 & 23,3 \\
\hline Estudante & 06 & 20,0 \\
\hline Procedência & & \\
\hline $\begin{array}{l}\text { Fortaleza } \\
\text { metropolitana }\end{array}$ & região & 16 & 53,3 \\
\hline Interior & & \\
\hline Dados da pesquisa & 14 & 46,7 \\
\hline
\end{tabular}

Dados da pesquisa

Quanto aos antecedentes obstétricos, na tabela 2, a maior parte das mães, 23 $(76,7 \%)$, realizaram seis consultas no pré-natal, predominantemente, em Unidades Básicas de Saúde (UBS), 21 (70\%). Os principais problemas ocorridos durante as gestações foram hipertensão arterial sistêmica, 11(36,6\%), e infecção do trato urinário 7 mães $(23,3 \%)$.

Tabela 2 - Condições da gestação e parto dos prematuros. Fortaleza-CE, 2015

\begin{tabular}{|c|c|c|c|}
\hline \multicolumn{4}{|c|}{ Variáveis maternas } \\
\hline $\begin{array}{l}\text { Duração } \quad \text { da } \\
\text { (semanas) } \leq 31\end{array}$ & 13 & 43,3 & \\
\hline 32 a 34 & 14 & 46,7 & $33,33 \pm 11,97$ \\
\hline \multicolumn{4}{|l|}{35 a 36} \\
\hline \multicolumn{4}{|l|}{ Tipo de gravidez } \\
\hline Única & 26 & 86,7 & \\
\hline Múltipla & 4 & 13,3 & \\
\hline \multicolumn{4}{|c|}{ Consulta Pré-natal } \\
\hline Até 6 & 23 & 76,7 & $5,0 \pm 2,0$ \\
\hline 35 a 36 & 7 & 23,3 & \\
\hline \multicolumn{4}{|l|}{ Tipo de parto } \\
\hline Normal & 12 & 40,0 & \\
\hline Cesáreo & 18 & 60,0 & \\
\hline \multicolumn{4}{|c|}{ Local do Pré-natal } \\
\hline UBS & 21 & 70,0 & \\
\hline Hospital & 08 & 26,6 & \\
\hline Outros & 01 & 3,3 & \\
\hline \multicolumn{4}{|c|}{ Problemas na gestação } \\
\hline HAS & 11 & 36,6 & \\
\hline ITU & 7 & 23,3 & \\
\hline Sangramento & 3 & 10,0 & \\
\hline Amniorrex & 1 & 3,3 & \\
\hline Sem alterações & 08 & 26,6 & \\
\hline
\end{tabular}

Dados da pesquisa. 
Na tabela 3, a maioria das mães, 18 (60\%), não teve experiência prévia com amamentação e 20 mães $(63,4 \%)$, não se sentiam seguras para amamentar. 0 primeiro momento em que as mães tocaram o filho, foi durante a troca da incubadora: 14 mães (46,6\%). Esse cuidado faz parte da rotina de prevenção e controle de infecção da unidade neonatal. O profissional que mais facilitou o primeiro contato do binômio-mãe-filho foi o técnico de enfermagem, o equivalente à resposta de 21 mães, (72,4\%). No que tange às dúvidas na alta hospitalar, a maioria das mães entrevistadas 18 mães (60\%) gostariam de saber mais sobre o banho. As dúvidas quanto à amamentação ocuparam o segundo lugar, sendo referidas por 17 participantes, $(56,7 \%)$. O engasgo foi a terceira dúvida mais frequente entre as mães.

Tabela 3 - Descrição das variáveis em relação ao contato, amamentação e orientação das mães de recém-nascidos internados na unidade neonatal. Fortaleza - CE, 2015.

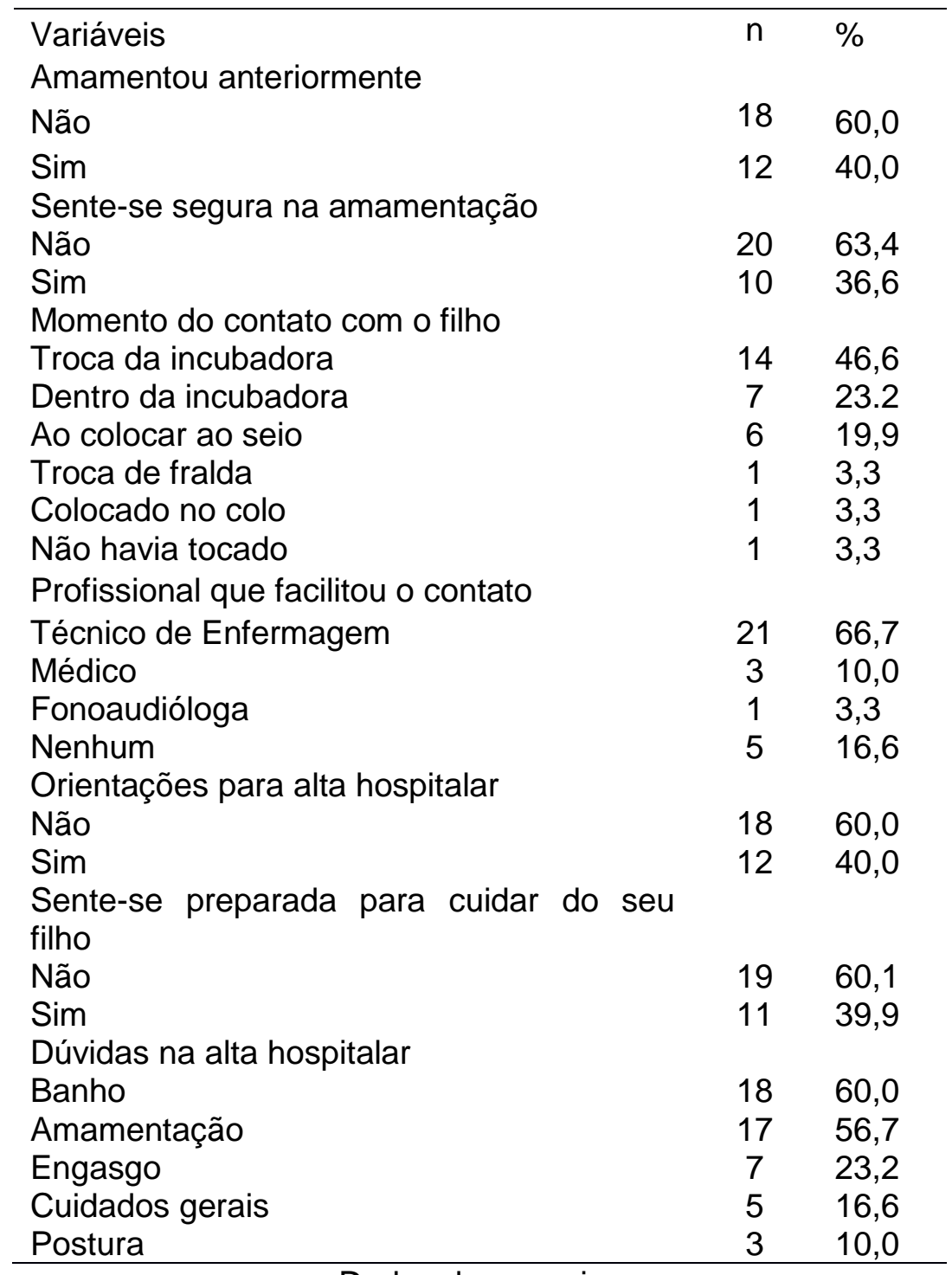

Dados da pesquisa

\section{DISCUSSÃo}


A caracterização das mães participantes, (Tabela 1), desta investigação, corrobora com pesquisas nacionais que descrevem faixa etária ente 20 e 35 anos, ensino médio e em união consensual (COUTO; PRAÇA, 2012; COSSUL et al. 2015; VERONEZ et al. 2017). Destacam-se o estudo de Couto e Praça (2012) realizado em São Paulo e que identificou o suporte materno para o cuidado do recém-nascido prematuro no domicílio. Já a pesquisa de Veronez et al. (2017), realizado na região sul, acrescenta além destas características, o histórico de aborto anterior das mães dos RN prematuros.

Sobre estes aspectos, Brocchi e Leme (2013) ao verificar o impacto da interação mãe-filho no desenvolvimento da linguagem oral de crianças prematuras, destacam a influência do nível socioeconômico da família e o estímulo a hábitos de leitura para o melhor o desenvolvimento da criança. Tais aspectos estão relacionados à idade, escolaridade e rede de apoio social da mãe cuidadora.

Nesse sentido, acredita-se que o cuidado ao RN prematuro deve incluir uma avaliação do enfermeiro no contexto hospitalar sobre as potencialidades e desafios da mãe na sua preparação para receber o filho de alta hospitalar. Por isso o conhecimento da caracterização socioeconômica é relevante para a sistematização do cuidado de enfermagem.

Alta hospitalar é um momento esperado pela mãe e familiares de prematuros, pois marca a saída do filho do ambiente hospitalar para o domicílio. É um momento de comemoração para algumas mães e medo e ansiedade para outras, que iram cuidar de seus filhos integralmente a partir desta saída. A pesquisa de Frota et al. (2013) descreve que o despreparo das mães interfere de forma negativa na saúde do bebê, e que o sentimento de exclusão na função materna provocados durante a internação pode repercutir no cuidado domiciliar do bebê.

O nascimento de prematuros ainda é um dos problemas que leva ao aumento da morbimortalidade. No estudo, demonstra-se que o número de consultas realizadas pelas mães no pré-natal foi seis ou mais (Tabela 2), podendo ser consideradas satisfatórias, comparando-se com a realidade nacional. Recomenda-se que as gestantes tenham pelo menos sete consultas de atendimento pré-natal. Em 2012, $62,4 \%$ das gestantes realizaram sete ou mais consultas pré-natal; $27,3 \%$ tiveram de quatro a seis consultas; 7,3\% tiveram de uma a três consultas; e, 3\% não tiveram nenhuma consulta pré-natal (BRASIL, 2015).

Sobre a ocorrência de partos cesáreos, a pesquisa segue os padrões do país e no mundo, ou seja, em 2010, enquanto as grandes regiões nacionais Nordeste e Norte apresentaram proporções de partos cesáreos de $41 \%$ e $44 \%$, respectivamente, o Sul e o Sudeste tiveram proporções mais elevadas, de $58,1 \%$ e $58,2 \%$, respectivamente (BRASIL, 2010). Em 2014, o Brasil apresentou uma taxa de 55\% cesáreas, uma das maiores no mundo, sendo relatado (WORLD HEALTH ORGANIZATION, 2015) que a taxa ideal de cesáreas seria entre $10 \%$ e $15 \%$ de todos os partos. 
A partir da Tabela 3, descreve-se a inserção da mãe na rotina de cuidados do RN prematuro: procedimentos estes que favorecem a interação mãe-bebê e promovem o vínculo. Pode-se citar como situações que promovem esta interação: amamentação, colocar ao seio e no colo, troca de fraldas. Destacam-se ainda a relação com a equipe hospitalar, com referência ao profissional que facilitou o contato (com maior prevalência o técnico de enfermagem), orientações para alta hospitalar favorecendo o preparo para cuidar do seu filho

A maioria das mães informou não ter experiências positivas quanto à amamentação em puerpérios anteriores, conforme descrito na Tabela 3. Tal fato mostra que a população envolvida no estudo apresenta fortes riscos de insucesso no aleitamento materno, o que deve ser motivo de maior atenção pela equipe de saúde. A experiência da amamentação é variável e depende do significado cultural que lhe é atribuído. Mães que foram amamentadas ou que viram outras mães amamentarem, têm uma representação positiva acerca da amamentação. Se as experiências transmitidas não foram positivas, essa pressão exercida sobre mãe pode contribuir negativamente para a sua experiência (HENRIQUE; MARTINS, 2011).

O aleitamento materno deve ser ensinado às mulheres/nutrizes, principalmente às mães de recém-nascidos prematuros, visto que são muitos os obstáculos presentes neste processo. O enfermeiro tem o papel de informar às famílias sobre os benefícios do leite materno, fornecer suporte e cuidado à mulher nutriz e seu filho, propor intervenções para obter uma lactação efetiva e fortalecer o vínculo familiar (BAPTISTA et al., 2015).

Na unidade, as mães tocaram em seus bebês pela primeira vez no momento em que o técnico de enfermagem fazia a troca rotineira da incubadora, momento oportunizado para o contato do binômio-mãe-filho. Para a construção do apego é necessário que a mãe possa tocar, acariciar, sentir o seu filho, pois essas reações aproximam o binômio-mãe-filho durante a hospitalização (OLIVEIRA; PAGLIUCA, 2015). Na unidade do estudo, o técnico de enfermagem foi quem mais promoveu momentos de contato entre mãe e filho.

Percebeu-se que as mães entrevistadas precisam de orientações para os cuidados domiciliares. Apesar de permanecerem boa parte do dia dentro da unidade, elas demonstraram insegurança para cuidar do filho após alta hospitalar. Cabem, aos profissionais de saúde que prestam assistência ao binômio, oferecerem as informações necessárias para o preparo da alta hospitalar. A mãe na unidade recebe muitas informações por ocasião da alta e muitas vezes não conseguem apreender tudo que lhe é transmitido em um curto espaço de tempo.

A importância da participação da mãe nos cuidados diários do seu bebê, na unidade neonatal, pode favorecer o vínculo mãe e filho. As mães podem superar 0 medo e a insegurança de cuidar do seu bebê, com o apoio dos profissionais de saúde que Ihe transmitem confiança e motivação no desempenho das atividades diárias 
(SANTOS et al., 2012). Nesse sentido, a capacitação da mãe para o cuidado domiciliar do prematuro, torna-se necessária durante todo o período de internação, pois assim, as habilidades para cuidar do bebê no domicílio poderão ser melhoradas.

A educação em saúde utilizada no processo de capacitação das mães oferece subsídios para o enfrentamento dos problemas e incrementa a sua formação social. $O$ conhecimento científico produzido na área da Enfermagem é intermediado pelos profissionais que fornecem informações com objetivo de alcançar a vida cotidiana dessas mulheres (LEITE et al., 2015).

A tabela 3 apresenta os resultados evidenciados sobre as dúvidas das mães quanto aos cuidados domiciliares com o recém-nascido, como o banho, a amamentação, o engasgo, os cuidados gerais e a postura. Dúvidas sobre o cotidiano de cuidados e que revelam a necessidade de maior aproximação e interação dos profissionais de saúde com os pais, para que essas informações sejam transmitidas e, com isso, a qualidade do cuidado prestado no hospital seja mantida (FROTA et al. 2013).

O banho foi à dúvida mais referida pelas mães. Elas têm receio de causar danos físicos ao bebê prematuro que é tão pequeno e frágil. Os temores mais frequentes citados foram o risco de queda e de entrada de água no canal auditivo. Sobre esse aspecto Veronez et al. (2017) destacam que o primeiro banho costuma gerar muitas expectativas, deixando a mãe e insegura. Os autores sugerem que as orientações dos cuidados delegados às mães devem ser repetidas para que sejam assimilados favorecendo a aquisição de segurança para a sua realização. O Enfermeiro deve inicialmente demonstrar, orientar, depois auxiliar e por último supervisionar a realização do procedimento.

A amamentação foi a segunda dúvida mais apontada pelas mães. As dúvidas estão relacionadas com a posição adequada, com a preocupação de ter leite suficiente e com a oferta de leite suplementar. O leite materno exclusivo é a alimentação indicada para recém-nascido, principalmente para o prematuro, segundo as recomendações da Organização Mundial de Saúde e o Ministério da Saúde do Brasil (BRASIL, 2011).

Outra dúvida citada, foi como proceder diante do engasgo. As mães sempre relatam a importância de serem capacitadas durante o período de internação de seus filhos, dando muita importância aos ensinamentos recebidos sobre os cuidados maternos no ambiente domiciliar (FROTA et al., 2013). O enfermeiro dentro do contexto hospitalar pode utilizar a presença da mãe e dos familiares na unidade neonatal como uma oportunidade para ensinamentos, que proporcionem mudanças positivas, amenizando estresses, potencializando a experiência com a construção de resultados que favoreça o binômio-mãe - filho (COUTO; PRAÇA, 2012). 
Nesse sentido, Cossul et al. (2015) reforçam a necessidade de acompanhamento sistematizado que veicule um cuidado especializado que considere as características e necessidades das crianças nascidas prematuras e de seus pais/familiares. Uma vez que a alta hospitalar é um momento importante para as famílias, pois é a concretização na maioria das vezes de um sonho da rede familiar (pais, irmãos, avós) receber o recém-nascido no domicílio, e a partir de então acompanhar seu crescimento e desenvolvimento.

\section{CONCLUSÃo}

Conclui-se que as mães apresentavam dúvidas quanto aos cuidados domiciliares do filho prematuro no preparo da alta hospitalar. Percebeu-se a insegurança da mãe para prestar os cuidados demandados pelo bebê ao chegar ao domicilio. As dúvidas relatadas estavam relacionadas aos cuidados rotineiros como banho, amamentação e engasgo.

A amamentação foi o segundo cuidado mais relatado. As mães precisam aprender o manejo adequado da amamentação, que inclui principalmente a posição correta, frequência e duração das mamadas, o que fazer quando o leite é insuficiente, entre outros.

A promoção do cuidado domiciliar do prematuro requer a participação de toda a equipe neonatal, em particular dos profissionais de enfermagem, que prestam assistência contínua. A mãe deve ser estimulada e apoiada para cuidar do bebê durante todo o período de internação. Desse modo se apropriará de meios adequados para cuidar do neonato com destreza e segurança.

\section{REFERÊNCIAS}

BAPTISTA, S. S. et al. Manejo clínico da amamentação: atuação do enfermeiro na unidade de terapia intensiva neonatal. Revista de Enfermagem UFSM, v. 5, n. 1, p. 23-31, 2015.

BOTÊLHO, S. M. et al. O cuidar materno diante do filho prematuro: um estudo das representações sociais. Revista da Escola de Enfermagem USP, v. 46, n. 4, p. 929934, 2012.

BRASIL. Ministério da Saúde. Departamento de Informática do Sistema Único de Saúde [Internet]. Brasília: Ministério da Saúde, 2010. Disponível em:

<http://www.datasus.gov.br>. Acesso em: 14 mar. 2017.

BRASIL. Ministério da Saúde. Atenção à Saúde do Recém-Nascido. Guia para os Profissionais de Saúde. Secretaria de Vigilância em Saúde. Brasília: Ministério da Saúde, 2011 (Série A. Normas e Manuais Técnicos).

BRASIL. Secretaria de Políticas para as Mulheres. Relatório Anual Socioeconômico da Mulher. Brasília: Secretaria de Políticas para as Mulheres, mar. 2015. 181 p. 
Disponível em: <http://www.spm.gov.br/central-deconteudos/publicacoes/publicacoes/2015/livro-raseam_completo.pdf>. Acesso em: 3 jan. 2017.

BROCCHI, B. S.; LEME, M. I. S. A relação entre a interação mãe-criança no desenvolvimento da linguagem oral de recém-nascidos prematuros. Audiology Communication Research, v. 18, n.4, p.321-31, 2013.

COSSUL, M. U. et al. Crenças e práticas parentais no cuidado domiciliar da criança nascida prematura. Revista Mineira de Enfermagem. Out/dez, v. 19, n. 4, p. 830835, 2015.

COUTO, F. F.; PRAÇA, N. S. Recém-nascido prematuro: suporte materno domiciliar para o cuidado. Revista Brasileira de Enfermagem, v. 65, n. 1, p. 19-26, 2012.

FONSECA, E. L.; MARCON, S. S. Percepção de mães sobre o cuidado domiciliar prestado ao bebê nascido com baixo peso. Revista Brasileira de Enfermagem, v. 64, n. 1, p. 11-17, 2011.

FROTA, M. A. et al. Alta hospitalar e o cuidado do recém-nascido prematuro no domicílio: vivência materna. Escola Anna Nery Revista de Enfermagem, v. 17, n. 2 , p. 277-283, 2013.

HENRIQUE, S. N.; MARTINS, R.M. L. Aleitamento materno: o porquê do abandono. Millenium, v. 40, p. 39-51, 2011.

LEITE, A. G. et al. Práticas de educação em saúde na Estratégia Saúde da Família: revisão integrativa da literatura. Revista de Enfermagem UFPE, Recife, v. 9, Supl. 10, p. 1572-1579, dez. 2015.

MELO, R. C. J.; SOUZA, I. E. O.; PAULA, C. C. O sentido do ser-mãe-que-tem-apossibilidade-de-tocar-o-filho-prematuro na unidade intensiva: contribuições para a Enfermagem neonatal. Escola Anna Nery Revista de Enfermagem, v. 16, n. 2, p. 219-226, 2012.

OLIVEIRA, P. M. P.; PAGLIUCA, L. M. F. Avaliação de tecnologia educativa na modalidade literatura de cordel sobre amamentação. Revista da Escola de Enfermagem USP, v. 47, n. 1, p. 205-212, 2013.

RAPOPORT, A.; PICCININI, C. A. Maternidade e situações estressantes no primeiro ano de vida do bebê. Psico-USF, Itatiba, v. 16, n. 2, p. 215-225, ago. 2011.

SANTOS, L. M. et al. Vivências paternas durante a hospitalização do recém-nascido prematuro na Unidade de Terapia Intensiva Neonatal. Revista Brasileira de Enfermagem, Brasília, v. 65, n. 5, p. 788-794, out. 2012.

SCHIMIDT, K. T.; HIGARASHI, I. H. Experiência materna no cuidado domiciliar do recém-nascido prematuro. Revista Mineira de Enfermagem, v. 16, n. 3, p. 391-399, 2012.

SIQUEIRA, M. B. C.; DIAS, M. A. B. A percepção materna sobre vivência e aprendizado de cuidado de um bebê prematuro. Epidemiologia e Serviços de Saúde, Brasília, v. 20, n. 1, p. 27-36, mar. 2011. 
VERONEZ, M.; et al. Vivência de mães de bebês prematuros do nascimento a alta: notas de diários de campo. Rev. Gaúcha Enferm., Porto Alegre, v. 38, n. 2, e60911, 2017.

WORLD HEALTH ORGANIZATION. Declaração da OMS sobre taxas de cesáreas. Brasília: Organização Mundial de Saúde, 2015. Disponível em:

<http://apps.who.int/iris/bitstream/10665/161442/3/WHO_RHR_15.02_por.pdf>. Acesso em: 14 mar. 2017. 